\title{
Holt-Oram Syndrome in a family from Eastern Nepal
}

Navaraj Paudel, ${ }^{a *}$ Nikesh Raj Shrestha, ${ }^{\text {b }}$ Puru Koirala, ${ }^{\mathrm{b}}$ Ram chandra Kafle, ${ }^{\mathrm{b}}$ Niraj Kumar Jaiswal, ${ }^{\mathrm{b}}$ Sanjeev Thapa ${ }^{\mathrm{c}}$

${ }^{a}$ Department of internal medicine, College of medical Sciences, Bharatpur, Nepal

${ }^{b}$ Department of internal medicine, BPKIHS, Dharan, Nepal

'Department of internal medicine, Patan Hospital, Kathmandu, Nepal

\section{Date of acceptance}

May 14th, 2012

\section{DOI Name}

10.3126/jaim.v1i2.6530

\section{Keywords \\ congenital heart defects, Holt-OramSyndrome, limb anomalies}

\section{Citation}

Paudel N, Shrestha NR, Koirala P, et al. Holt-Oram Syndrome in a family from Eastern Nepal. Journal of Advances in Internal Medicine 2012;01(2):70-2.

\section{ABSTRACT}

Holt-Oram Syndrome is an autosomal dominant disorder with complete penetrance characterized by congenital cardiac defects and skeletal abnormalities of the upper limbs. Till now more than 300 cases have been reported showing wide spectrum of clinical signs but to the best of our knowledge, reporting of familial cases from Nepal has not been done. We found Holt-Oram syndrome in a 16 years old male patient with upper limb anomalies who was being evaluated for dyspnea and palpitation. Similar findings were later found in his two siblings. We recommend a thorough physical and cardiac evaluation for patients with limb anomalies who present with cardiac symptoms or history of recurrent pneumonias.

\section{INTRODUCTION}

Holt and Oram first described a family with Atrial Septal Defect (ASD) and thumb abnormalities in $1960 .{ }^{1}$ This combination of cardiac abnormalities and skeletal deformity is now commonly known as Holt Oram Syndrome (HOS) or sometimes as Heart Hand Syndrome. Today it is known to be a rare genetic disorder with the incidence of around 1 in 1,00,000 live births. $^{2}$

About $75 \%$ of people with HOS have cardiac problems which can be life threatening. ${ }^{3}$ Early diagnosis and treatment helps to improve quality of life and to increase longevity of affected individuals. To the best of our knowledge no cases of HOS have been reported from Nepal till date. Here we present 3 siblings from a family from Jhapa district of eastern Nepal with HOS.

\section{CASE}

A 16 years old male presented to medical OPD with history of insidious onset gradually progressive breathlessness and palpitation since 7 years This was associated with history of recurrent pneumonias since childhood. He had small hands with deformed fingers and palm since birth. He had otherwise normal developmental milestones. He has an elder sister of 21 years and a younger brother of 11 years with similar complaints. His parents had no such complaints. On examination the vital parameters and intellect were normal. There was high arched palate, small hands and fingers with absent thumbs bilaterally. Pectus excavatum was present. These findings were uniformly present in both of his siblings.

On cardiovascular examination, the apex was localized normally; pulmonic component of second heart sound was palpable with systolic thrill in pulmonary area. Left para-sternal heave of AIIMS grade III suggestive of right ventricular (RV) enlargement was present. The second heart sound was widely split and fixed. There was ejection systolic murmur in the pulmonary area. The findings were similar in the others although the severity was slightly variable. On investigation, blood parameters were normal; Chest X-ray showed cardiomegaly suggestive of RV enlargement, electrocardiography showed right axis deviation, first degree heart block, complete right bundle branch block and p pulmonale. Trans thoracic echocardiography showed dilated right atrium and right ventricle, Ostiumsecondum type of ASD. The other two siblings were screened and results were similar. USG abdomen and X-ray spine to look for other anomalies were done which was normal.

In view congenital heart disease (ostium secondum type of ASD) with upper limb anomalies (triphalangeal fingers with absent thumbs) the diagnosis of "Holt-Oram Syndrome" was made .

\section{DISCUSSION}

HOS is an autosomal dominant disease characterized by skeletal abnormalities of the hands and arms (upper limbs) and heart problems. Most cases result from new mutations in the gene and occur in people with no history of the disorder in their family. Contributory environmental factors are not known. ${ }^{4}$ But there are mutations in the TBX5 gene causing this disease. Linkage studies demonstrate that the gene defect resides on the long arm of chromosome $12 .{ }^{5}$ Although the clinical manifestations are variable, upper limb abnormalities are always present. Abnormalities may be unilateral or bilateral and symmetric or asymmetric and may involve the radial, carpal, and thenar bones. Aplasia, hypoplasia, fusion, or anomalous development of these bones produces a spectrum of phenotypes, including triphalangeal or absent thumbs with the most prevalent findings being malformations or fusions of the carpal bones. ${ }^{2,4-6}$ Rarely it is associated with foot anomalies also. ${ }^{7}$ Similarly in our cases, the patients had bilateral small/hypoplastic hands with absence of thumbs which is similar to description as in typical cases reported earlier.

\footnotetext{
* Corresponding author Navaraj Paudel,

Department of Medicine, College of medical Sciences, Bharatpur, Nepal Email address: -drnavarajmd@gmail.com
} 


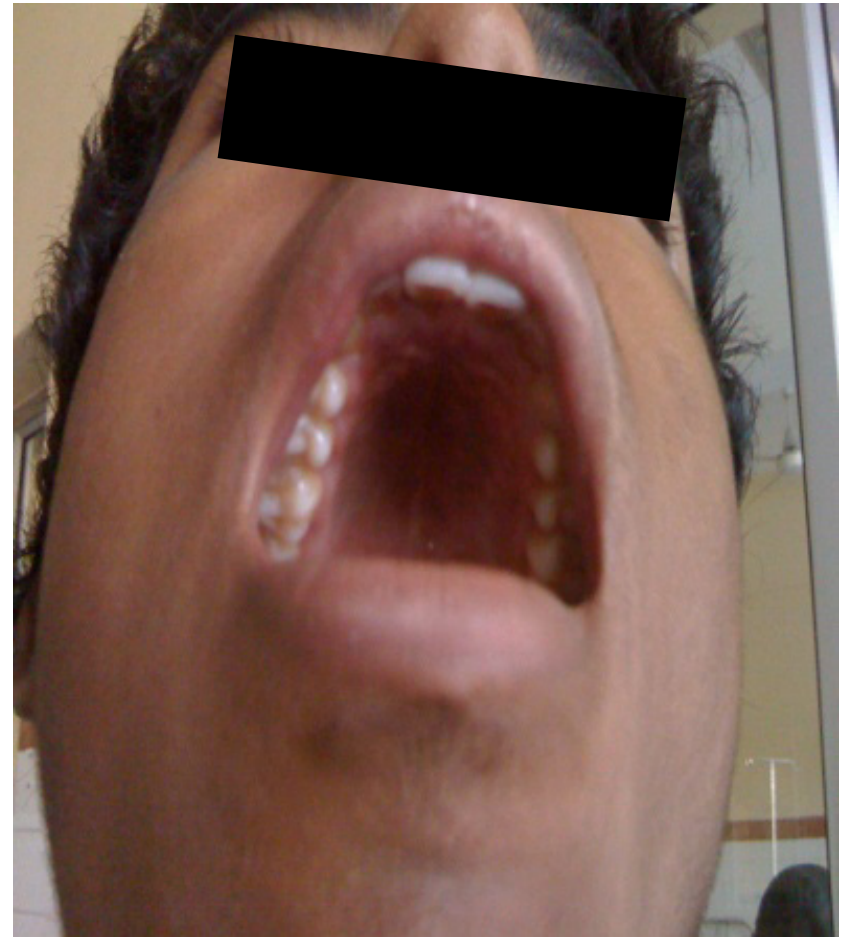

Figure 1. High arched palate

Approximately $75 \%$ of patients have some cardiac abnormality. ${ }^{2}$ In most patients, the abnormality is an ASD and rarely VSD, which varies in number, size, and location. ASDs are usually of the secundum variety, while VSDs tend to occur in the muscular trabeculated septum. ${ }^{2,4-6}$ Similarly in our cases we found the ostiumsecundum type of ASDs although the size and location varied in all three cases. Other cardiac anomalies also may include cardiac conduction defects such as AV blocks and $\mathrm{AF}^{2,4}$ Cardiac conduction defect is progressive with aging. Sometimes the first clinical manifestation of the disease may be heart failure, cardiac arrhythmias (including heart block), or infective endocarditis. ${ }^{2}$ The Electrocardiographic abnormality found in our cases was the complete right bundle branch block with first degree AV block. The features of HOS are similar to those of a condition called Duane-radial ray syndrome; however, these two disorders are caused by mutations in different genes. ${ }^{\text {? }}$

Diagnosis is done by clinical features, radiography of the hands including wrist joint showing abnormal carpel bones, absent or abnormal radial bone with various displacement deformities. ${ }^{2}$ Chest radiography may demonstrate enlarged pulmonary arteries due to pulmonary hypertension or cardiomegaly with evidence of congestive heart failure as in our case we found that there was apparent cardiomegaly of right ventricular type with features of mild pulmonary artery hypertension. Wrist radiography of the parents of the patient with HOS to establish a familial versus sporadic nature of the syndrome in the family should be considered. Echocardiography is the procedure of choice to define the presence of septal defects or other cardiac anomalies. ${ }^{2}$ An ECG is done to find out the involvement of the conduction system. ${ }^{2,8}$ If suspicion of intermittent dysrhythmia, 24hour holter monitoring may be useful. Knowing the progressive nature of this disease, periodic evaluation for conduction system involvement, even in the absence of cardiac structural disease, is important. ${ }^{9}$

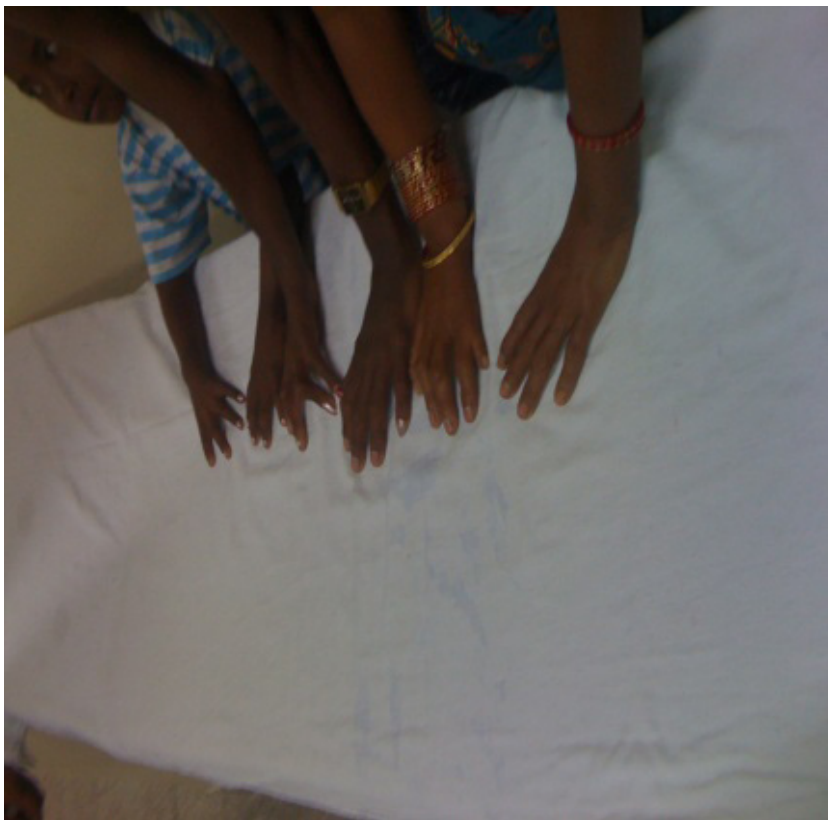

Figure 2. Triphalangeal fingers of three siblings

Genetic evaluation is important. ${ }^{10-12}$ Mutational analysis of TBX5 is not available on a routine clinical basis and remains a research tool. TBX5 mutations are detected in about $75 \%$ of individuals meeting strict clinical criteria for HOS. ${ }^{2}$ Identification of the disease causing mutation in a family may allow for pre-implantation genetic diagnosis for co-couples at $50 \%$ risk of having an affected child. ${ }^{9,-11}$ The genetic diagnosis in our cases could not be performed due to lack of the resources in our set up.

Management is mostly by proper evaluation and regular follow up. The patients with high risk for Eisenmenger syndrome should be evaluated by cardiac catheterization to define the nature and severity of intra-cardiac shunts as they may require surgical intervention. ${ }^{2}$ Evaluation can usually be performed in an outpatient setting. Physical activity is limited if heart failure or persistent cardiac sequel are present. ${ }^{2}$ Medications are not effective in treating the anatomical defects of patients with HOS. Anticoagulation should be considered in patients with pulmonary hypertension and cardioversion, anti-arrhythmic drug therapy, or anticoagulation should be considered in patients with $\mathrm{AF}^{2,{ }^{2,3}}$ Antibiotic prophylaxis should be applied following standard American Heart Association/American College of Cardiology guidelines for patients with congenital heart disease. ${ }^{2}$ Inpatient studies and surgical treatment may be necessary for the patients with advanced heart block that may require a permanent pacemaker and surgical therapy to correct cardiac defects or to improve limb function. ${ }^{2}$ Septal defects with normal hemodynamic shunts do not require correction. Children with severe limb anomalies can be referred to orthopedic surgeons and may benefit from prostheses. Complications known are congestive heart failure, arrhythmias like heart blocks, AF, infective endocarditis, Sudden death etc. ${ }^{2,3}$ Prognosis is generally good, but it depends on the severity of the cardiac malformations. Periodic follow-up for cardiac conduction disease is warranted in all affected individuals at least annually. ${ }^{2}$ 


\section{CONCLUSION}

Patients with upper limb anomalies and cardiac diseases should be evaluated further to rule out HOS. Familial screening should be done. Though genetic diagnosis may not always be possible in resource poor settings like ours howeverearly diagnosis and intervention as required will definitely help to prevent the possible complications in such patients.

\section{ACKNOWLEDGEMENT}

Author is grateful for Dr. Prakash Thapa, Lecturer, Department of Psychiatry, Manipal College of Medical Sceinces, Pokhara, Nepal.

\section{REFERENCES}

1. Holt M, Oram S. Familial heart disease with skeletal malformations. Br Heart J 1960;22:236-4.

2. Warnes CA, Williams RG, Bashore TM, et al. ACC/AHA 2008 Guidelines for the Management of Adults with Congenital Heart Disease: Executive Summary: a report of the American College of Cardiology/American Heart Association Task Force on Practice Guidelines (writing committee to develop guidelines for the management of adults with congenital heart disease). Circulation 2008;118:2395-451.

3. McDermott DA, Hatcher CJ, Basson CT. Atrial Fibrillation and Other Clinical Manifestations of Altered TBX5 Dosage in Typical Holt-Oram Syndrome. Circ Res 2008;103:e96.

4. Basson CT, Huang T, Lin RC, et al. Different TBX5 interactions in heart and limb defined by Holt-Oram syndrome mutations. Proc Natl Acad Sci U S A 1999;96:2919-24.

5. Basson CT, Cowley GS, Solomon SD, et al. The clinical and genetic spectrum of the Holt-Oram syndrome (heart-hand syndrome). $N$ Engl J Med 1994;330:885-91.
6. Garavelli L, De Brasi D, Verri R, et al. Holt-Oram syndrome associated with anomalies of the feet. Am J Med Genet A 2008;146:1185-9.

7. Basson CT, Solomon SD, Weissman B, et al. Genetic heterogeneity of heart-hand syndromes. Circulation 1995;91:1326-9.

8. Cerbai E, Sartiani L. Holt-oram syndrome and atrial fibrillation: opening the (T)-box. Circ Res 2008; 102:1304-6.

9. Postma AV, van de Meerakker JBA, Mathijssen IB, et al. A Gain-of-function TBX5 mutation is associated with atypical Holt-Oram syndrome and paroxysmal atrial fibrillation. Circ Res 2008;102:1433-42.

10. Pete B, Harmath A, Szigeti Z, et al. Holt-Oram syndrome: genetic counseling and diagnosis with prenatal ultrasonography. Orv Hetil 2007;148:2173-6.

11. Sunagawa S, Kikuchi A, Sano Y, et al. Prenatal diagnosis of HoltOram syndrome: role of 3-D ultrasonography. Congenit Anom (Kyoto) 2009;49:38-41.

12. He J, McDermott DA, Song Y, et al. Preimplantation genetic diagnosis of human congenital heart malformation and Holt-Oram syndrome. Am J Med Genet 2004;126:93-8.

\section{ANSWER}

Inspection of chest shows inward depression of chest producing sunken in and caved appearance consistent with the diagnosis of pectus excavatum (Figure 1). Lateral CXR shows inward displacement of sternum with ribs seen anterior to sternum, suggests the diagnosis of pectus excavatum (Figure 2). CT scan thorax shows inward displacement of sternum compressing the heart, confirms the diagnosis of severe pectus excavatum with surgical indication (Figure 3 ).

Pectus excavatum is characterized by inward displacement of the sternum, creating a depression in the chest. Classification of pectus excavatum is categorized according to severity (mild, moderate, or severe) base on radiological criteria. The CT scan is used to ascertain the severity of the deformity, by determining the Haller index: a ratio of the measure of the transverse diameter of the chest, divided by the sagital measure of the distance from the sternum to the vertebral body. The Haller index should be obtained at the deepest point of the deformity. Haller index between 2 and 3.2 is considered a mild deformity; between 3.2 and 3.5 is moderate and 3.5 or above is a severe deformity. ${ }^{1}$ Both moderate and severe deformities can be considered candidates of corrective surgery. This patient had a Haller index of 3.90. The severity of this index, in the setting of PFTs consistent with restrictive pulmonary process, with an echocardiogram that reveals compression of the heart makes an indication of corrective surgery. A recent study revealed positive correlations between severity of sternal deformity and cardiac rotation, as well as clinically significant symptoms. ${ }^{2}$ The case was atypical in the sense that the symptoms of the patient did not correlate with the radiological findings.

\section{REFERENCES:}

1. Sidden CR, Katz ME, Swoveland BC, et al. Radiologic considerations in patients undergoing the Nuss procedure for correction of pectus excavatum. Pediatric Radiology 2001; 3:429-34.

2. Chu ZG, Yu JQ Yang ZG, et al. Correlation between sternal depression and cardiac rotation in pectus excavatum: Evaluation with helical CT. Am J Roentgenol 2010;195:76-80. 